\section{Examination of Metals by Polarized Light}

RECENTLy, several papers have been published on the use of polarized light for revealing the grain structure of metals and alloys. Hone and Pearson ${ }^{1}$ showed that by examining under polarized light a polished aluminium surface which had been anodized in a suitable electrolyte, the grain structure was revealed, differently oriented grains appearing with different brightnesses. Woodward ${ }^{2}$, by etching 'Monel' metal in a specific reagent, has found similar effects. Polished surfaces of aluminium and 'Monel' metal, being optically isotropic, do not react to polarized light as do the optically anisotropic surfaces of tin, zinc, cadmium, etc., and the above authors ${ }^{1,2}$ have attributed the optical anisotropy of the anodized aluminium and etched 'Monel' metal to the anisotropic properties of the surface film formed during anodizing or etching. Although this explanation may be correct, there is a possible alternative which involves the surface contour of the specimen, for it is well known that if pearlite is examined under polarized light no reaction occurs unless the specimen is etched. This has been shown convincingly by Baeyertz $z^{3}$ to be due to the presence of fine parallel grooves on the surface, formed by etching away the ferrite and leaving the cementite lamellæe in relief. Furthermore, Jones ${ }^{4}$ has shown that polarization effects can be produced by deeply etching a metal surface.

To decide which of these two explanations is correct, electrolytically polished zinc and cadmium, aluminium electrolytically polished and anodized in a solution containing 49 per cent water, 49 per cent ethyl alcuhol and 2 per cent hydroHuoric acid at 30 volts, and 'Monel' metal etched according to Woodward ${ }^{2}$, were examined before and after evaporating a silver film, approximately $800 \mathrm{~A}$. thick, on to the prepared surfaces. If the polarization effects are due to optical anisotropy, the silver, being optically isotropic, should destroy them: whereas, if they are due to the surface contour, they should still remain after silvering, for it is known that evaporated silver takes up the surface contour of

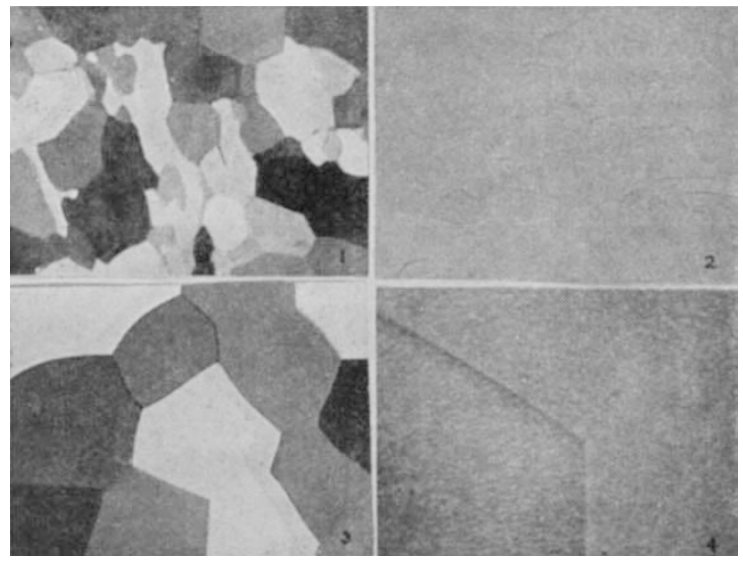

Fig. 1. Electrolytically polished cadmium : crossed nicols. $\times 125$ Fig. 2. Same as Fig. 1 with silver film present : crossed nicols.

Fig. 3. Polished and anodized aluminjum with silver flim present crossed nicols. $\times 20$

Fig. 4. Same as Fig. 3 photographed under phase-contrast the surface on which it is deposited (that is, any grooves present before silvering will also be present after).

Without the silver film, all four surfaces reacted to polarized light, a typical example being shown in Fig. 1. After silvering, the zine and cadmium showed no polarization effects (see Fig. 2), whereas both the etched 'Monel' metal and anodized aluminium still reacted to polarized light after deposition of the silver film (see Fig. 3). Similar results were also obtained on aluminium which was electrolytically polished and anodized in the way described by Hone and Pearson ${ }^{1}$. It is clear, therefore, that the polarization effects obtained with anodized aluminium and etched 'Monel' metal are due to some surface structure and not to true optical anisotropy. The silvered aluminium and 'Monel' metal surfaces were examined under the phase-contrast microscope, which is very sensitive in revealing small differences of level, to discover if some such structure could be seen. A structure was clearly seen on the aluminium (see Fig. 4), and faint evidence of one on the 'Monel' metal. Electron microseopic examination of the 'Monel' confirmed that a very fine structure was present. That the structure shown in Fig. 4 is due to the anodized aluminium surface and not to the silver alone is shown by the fact that a silver film evaporated on to an electrolytically polished aluminium surface showed no markings when examined under the phasecontrast microscope.

Further support for these conclusions was found in the observation that if polished aluminium is anodized in a solution containing 12 per cent disodium acid phosphate and 0.4 per cent sulphuric acid in water, which according to Edwards and Keller ${ }^{5}$ gives a structureless oxide coating as seen by the electron microscope, no polarization effects are observed.

We thank the Director and Council of the British Non-Ferrous Metals Research Association for permission to publish this communication.

\section{E. C. W. Perryman \\ J. M. LACK}

British Non-Ferrous Metals

Research Association,

Euston Street,

London, N.W.1.

Nov. 9.

1 Hone, A., and Pearson, E. C., Metal Progress, 53 (3), 363 (1948)

Woodward, D. H., J. Metals, 1 (10), 722 (1949).

saeyertz, M., Trans. Amer. Inst. Min. Met. Eng., 117, 151 (1935).

4 Jones, O., Phil. Mag., 48, 207 (1924).

${ }^{5}$ Edwards, J. D., and Keller, F., Metals Tech., 11 (3) (1914). Tech. Pub. No. 1710 .

\section{Source of the Light recorded in Photographs of Detonating Explosives}

IT is now generally recognized that a large part of the light arising from the detonation of $a$ condensed explosive cartridge is due to the system of shock waves produced in the surrounding air. When the air is replaced, for example, by water, the total luminosity is greatly reduced. The wave-speed photographs shown in Fig. 1 illustrate this effect. 